\title{
A pitfall in the cytodiagnosis of sputum of asthmatics
}

\author{
BERNARD NAYLOR AND CONSTANTINE RAILEY ${ }^{1}$ \\ From the Department of Pathology, the University of Michigan, \\ Ann Arbor, Michigan, U.S.A.
}

SYNOPSIS The sputum of an asthmatic often contains large, well-circumscribed clusters of benigri columnar epithelial cells which can be misinterpreted as papillary fragments of an adenocarcinoma $\vec{V}$ Their presence is a manifestation of the excessive shedding of the mucosa of the lower respiratorytract accompanying an attack of bronchial asthma. These clusters are illustrated and compare $0^{\circ}$ with clusters of adenocarcinoma cells. To discriminate between the two types of cluster may be difficult and it is therefore important for the cytologist to know when a sputum is from an asthmatic:-

It is not generally realized that the sputum of an asthmatic may harbour a serious pitfall for those who practice cytodiagnosis. In fact, the first falsepositive report in respiratory cytology from this laboratory was given on the sputum of an asthmatic. Other authors (Philps, 1956; Farber, Wood, Pharr, and Pierson, 1957; Bamforth, 1959; Cohen and Prentice, 1959) have reported similar difficulty. This problem arises from the fact that one of the pathological changes in bronchial asthma is the excessive shedding of the mucosa of the lower respiratory tract, a fact which may manifest itself in smears of sputum as compact clusters of columnar epithelial cells resembling papillary fragments of adenocarcinoma. In this paper we shall illustrate examples of the clusters found in sputa of asthmatics and, for comparison, those found in the sputa of patients with pulmonary adenocarcinoma.

\section{MATERIAL}

This study is based on the examination of sputa of 100 asthmatics, and of patients with pulmonary adenocarcinoma, including the multifocal variant, bronchiolarcell carcinoma. (Cells exfoliated from localized adenocarcinoma and from bronchiolar-cell carcinoma are morphologically the same. To maintain brevity the term 'adenocarcinoma' will cover both types of neoplasm.) None of the asthmatic patients had evidence of calcinoma and none of the patients with carcinoma were asthmatic. Smears were prepared and stained in the manner used in our laboratory for cytodiagnosis (Vincent Memorial Hospital, 1950).

${ }^{1}$ Papanicolaou Cancer Research Institute, 1155 N.W. 14th Street, Miami 36, Florida, U.S.A.

Received for publication 7 May 1963

\section{OBSERVATIONS}

Clusters in the sputa of asthmatics may be few but are usually numerous (Fig. 1). Their size varies: greatly (Figs. 2 and 3). Their shapes are commonlys oval (Fig. 2), but some are bizarre with animacula? outlines (Figs. 3 to 5). Most clusters are strikingly compact (Figs. 2 to 5), and many show neat palisad $\bar{D}$ ing of peripheral cells (Figs. 2 to 5), but, if any cells are enlarged by vacuolation (Figs. 6 to 8), the clusters may appear distended and rather dis organized.

In general, cell size and nuclear characteristics (size, shape, chromatin pattern, and staining reaction) are within normal limits for respiratory epithelium (Figs. 8 and 9). In about 1,300 clusters examined, no mitotic figures were noted.

As might be expected, many clusters bear cilia but there are others which seem devoid of them. In any smear which contains many clusters, cilia (Fig. 10) can usually be found on some of then? without difficulty; occasionally examination with an oil-immersion lens is necessary to confirm theip presence.

Almost all of the smears of the asthmatics' sput containing these columnar cell clusters also containo a large number of eosinophil leucocytes (Figs. 2 to 7) With the Papanicolaou stain they may be more readily recognized by their bilobed nuclei rather tharef by their faintly stained eosinophilic granules. Ino about one-half of cases of asthma, the smears o $\bar{B}$ sputum demonstrate well-formed Curschmann' spirals; a few smears contain Charcot-Leydero crystals. 


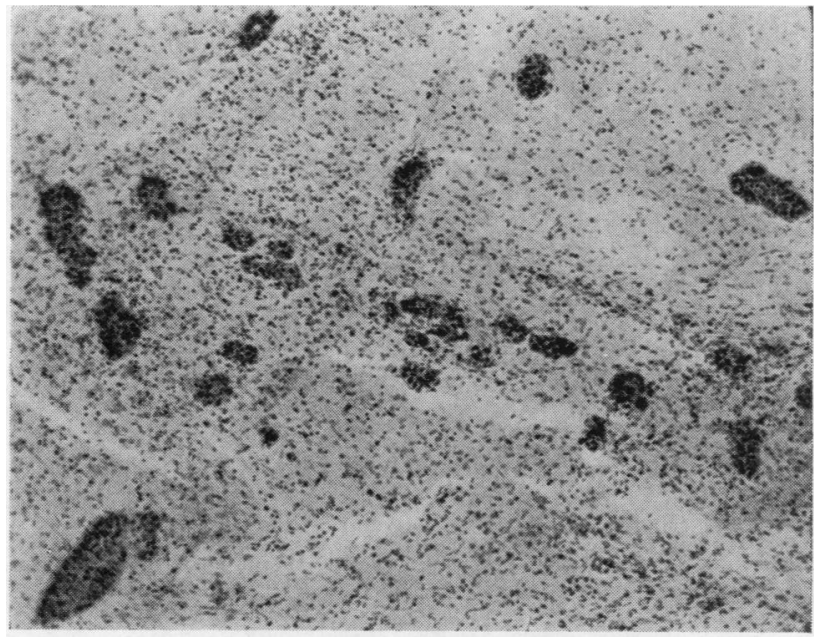

FIG. 1. Smear of an asthmatic's sputum showing numerous clusters of columnar epithelial cells. Papanicolaou $\times 76$.

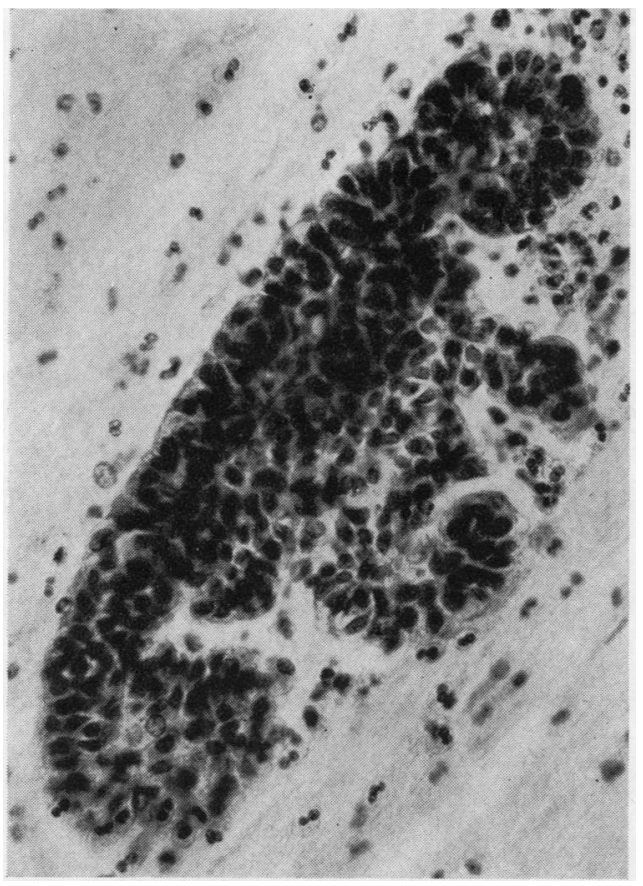

FIG. 3

FIGS. 3 to 5. Smears of an asthmatic's sputum. Large, compact, smoothly outlined, animacular clusters of columnar epithelial cells showing peripheral palisading. Note the presence of bilobed eosinophil leucocytes. Papanicolaou $\times 300$.

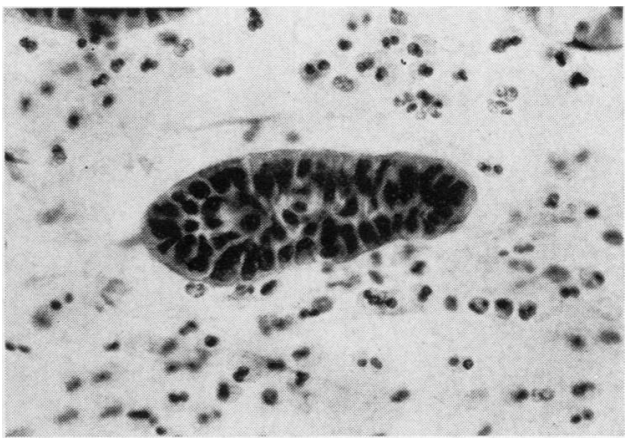

FIG. 2. Smear of an asthmatic's sputum. An oval, compact, medium-sized, smoothly outlined cluster of columnar epithelial cells showing peripheral palisading. In the surrounding mucus are many eosinophil leucocytes with bilobed nuclei. Papanicolaou $\times 300$.

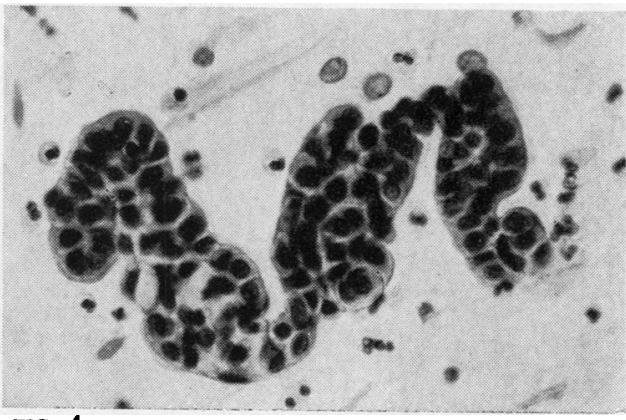

FIG. 4

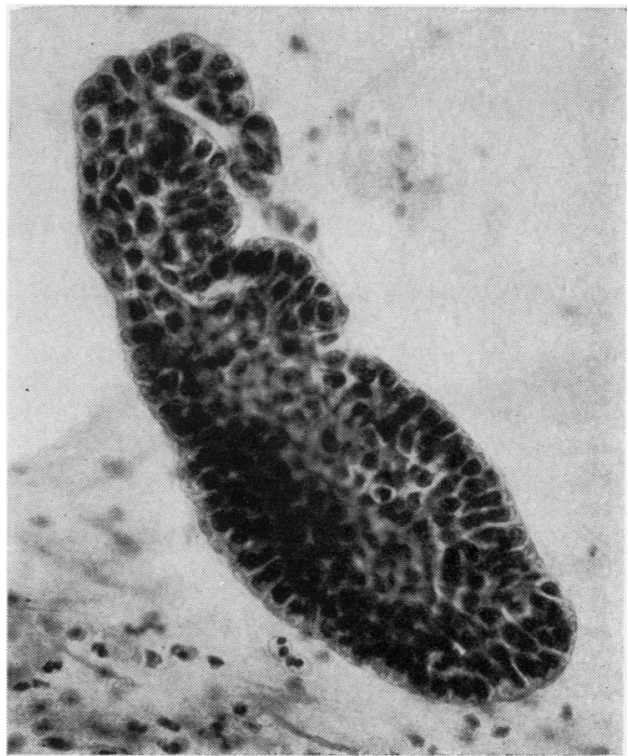

FIG. 5 


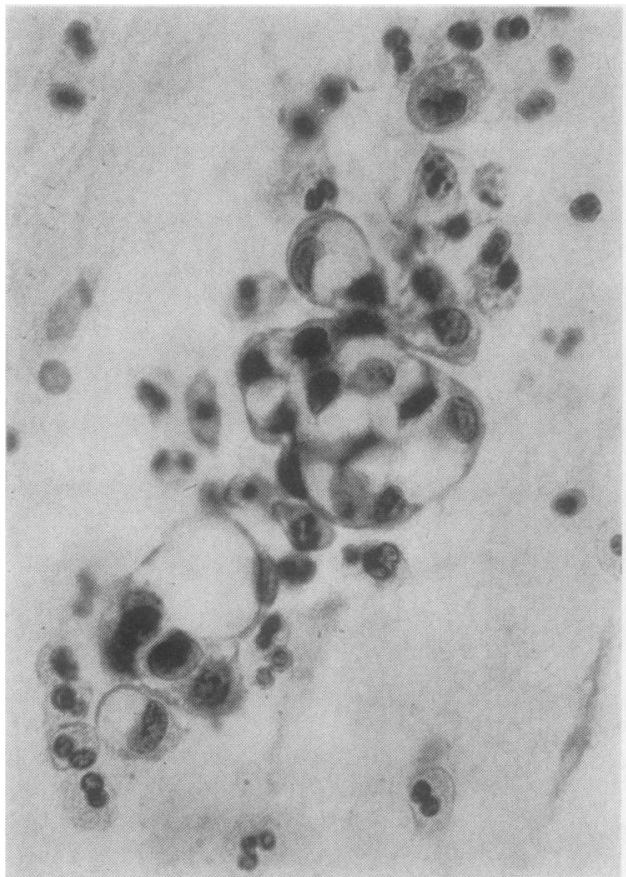

FIG. 6

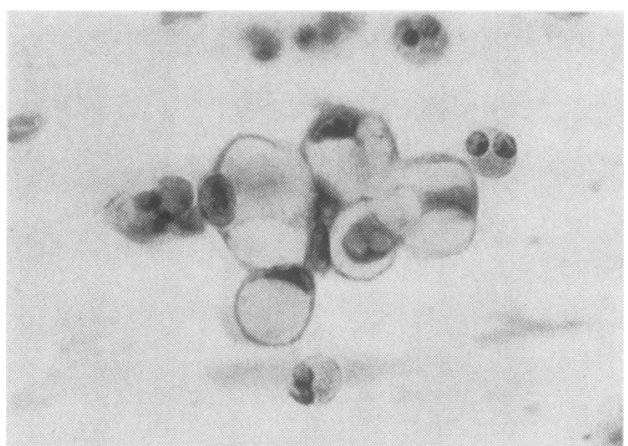

FIG. 7

FIGS. 6 and 7. Smears of asthmatics' sputa. Clusters of columnar epithelial cells appearing distended and rather disorganized by vacuolation. Both have a knobby outline. Note the presence of bilobed eosinophil leucocytes. Papanicolaou $\times 600$.

FIG. 8. Smear of an asthmatic's sputum. A cluster of columnar epithelial cells which appears rather disorganized by vacuolation. Cell size and nuclear characteristics are within normal limits for respiratory epithelium. Papanicolaou $\times 600$.

FIG. 9. Smear of an asthmatic's sputum. Enlargement of Fig. 4 to show that cell size and nuclear characteristics are within normal limits for respiratory epithelium. Papanicolaou $\times 600$.

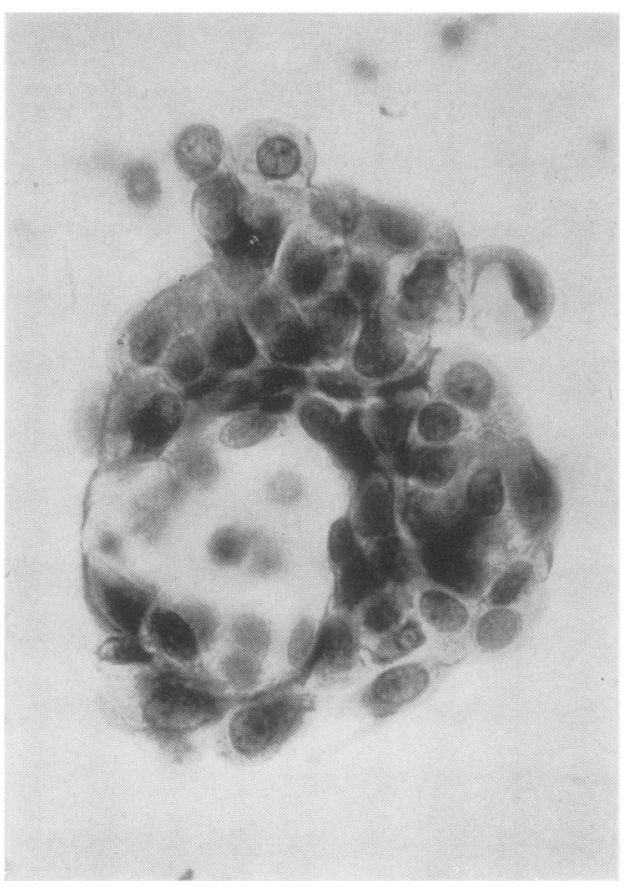

FIG. 8

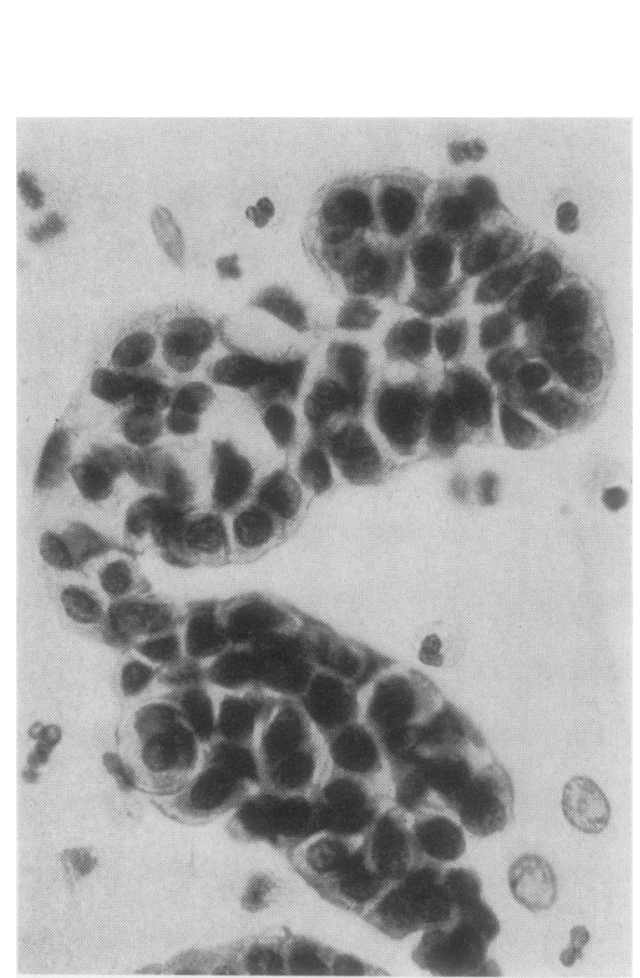

FIG. 9 


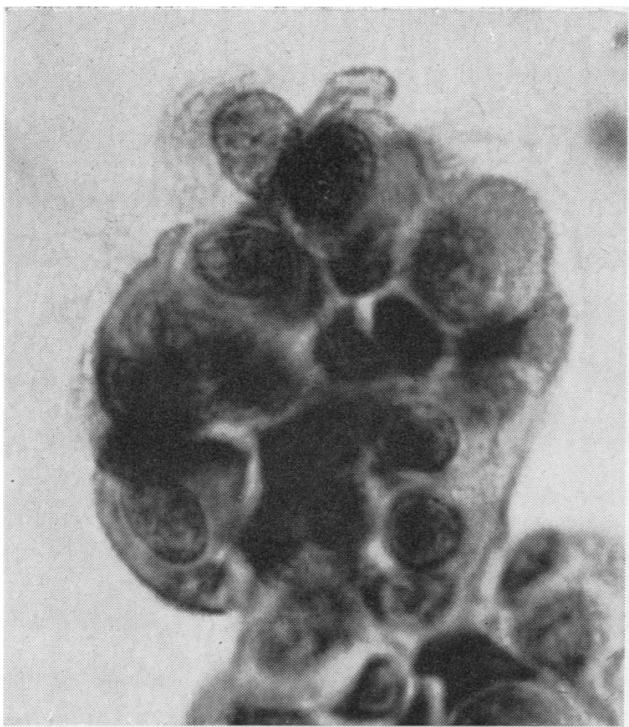

FIG. 10

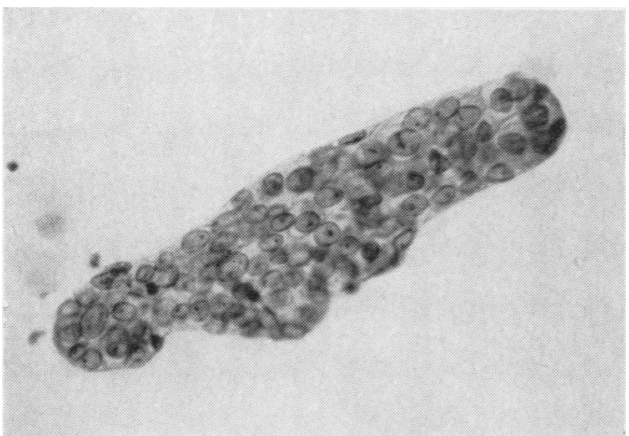

FIG. 14

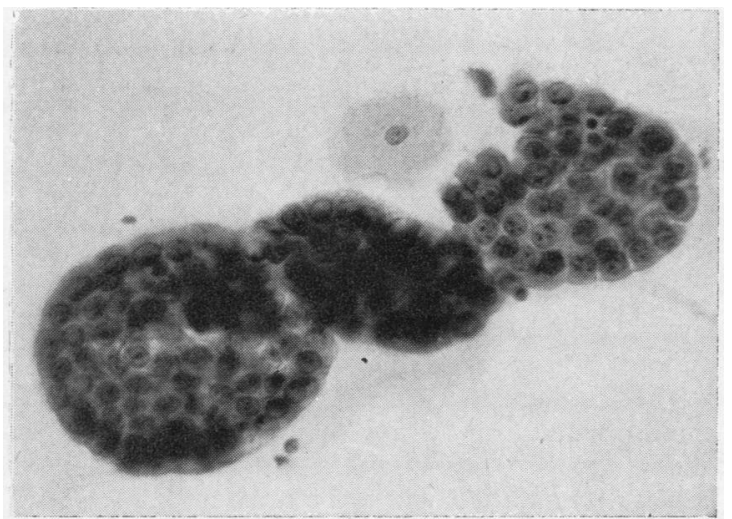

FIG. 15

FIGS. 14 and 15. Smears of sputum. Smoothly outlined, unusually shaped clusters of adenocarcinomatous cells. Figure 15 shows peripheral palisading. Papanicolaou $\times 300$.

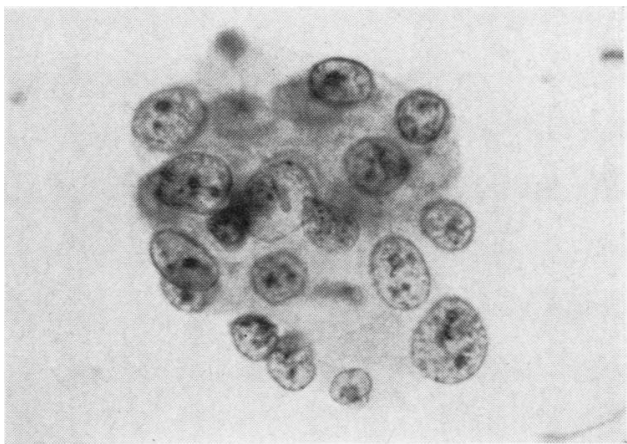

FIG. 11

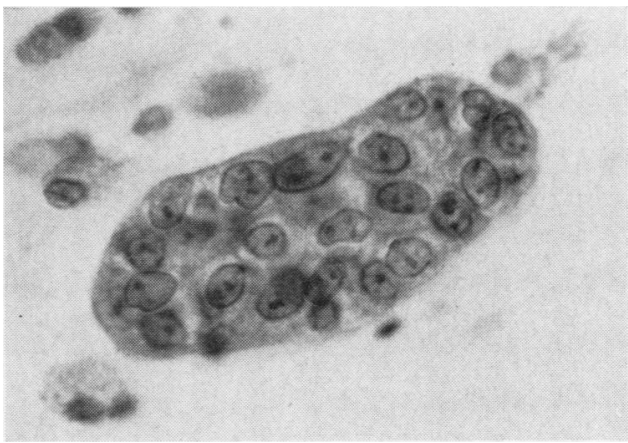

FIG. 12

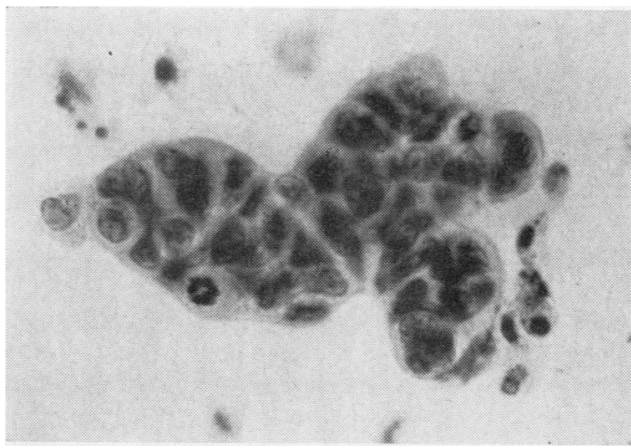

FIG. 13

FIG. 10. Smear of an asthmatic's sputum. Part of a cluster of columnar epithelial cells showing cilia. Papanicolaou $\times 1,200$.

FIG. 11. Smear of sputum. A cluster of adenocarcinomatous cells. It is nearly round, not too compact, has a knobby outline, and the nuclei appear malignant. Papanicolaou $\times 600$.

FIG. 12. Smear of sputum. Smoothly outlined, oval cluster of adenocarcinomatous cells. The nuclei appear malignant. Papanicolaou $\times 600$.

FIG. 13. Smear of sputum. An unusually shaped cluster of adenocarcinomatous cells. One cell shows a mitotic figure. Papanicolaou $\times 600$. 


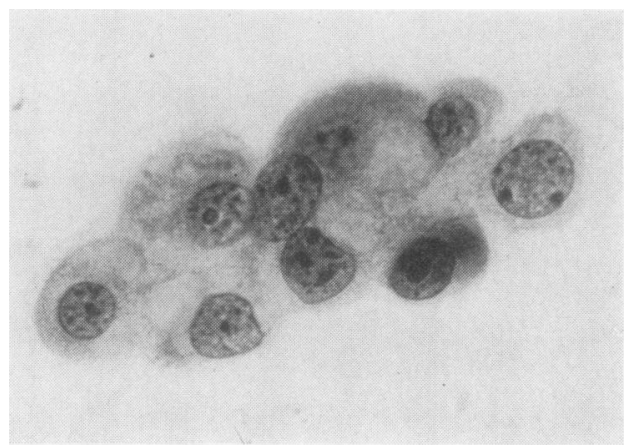

FIG. 16

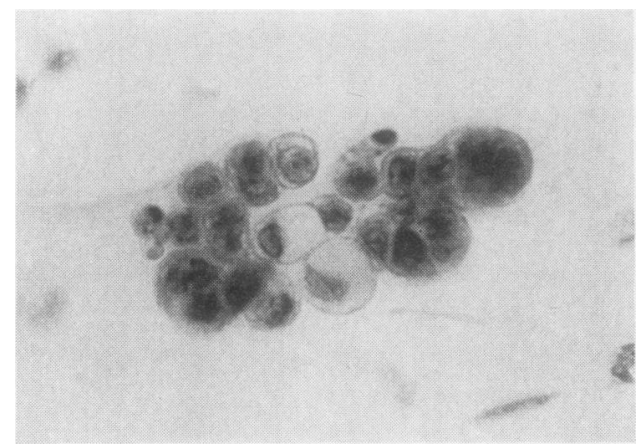

FIG. 17

FIG. 16. Smear of sputum. A cluster of adenocarcincmatous cells. It is not too compact, has a knobby outline, and the nuclei appear malignant. Papanicolaou $\times 600$.

FIG. 17. A cluster of adenocarcinomatous cells a few of which are vacuolated. Its outline is knobby. Papanicolaou $\times 600$.

FIGS. 18 and 19. Smears of sputa. Clusters of adenocarcinomatous cells. The clusters have a knobby outline. Most of the cells are vacuolated. In Fig. 18 many nuclei show an irregular chromatin pattern. Papanicolaou $\times 600$.

Figures 11 to 19 show clusters of exfoliated cells found in smears of sputum of patients with primary or metastatic pulmonary adenocarcinoma. The similarity between these clusters of neoplastic cells and the clusters found in asthmatics' sputa is apparent, but the differences between them are not always obvious. Clusters of carcinoma cells are not generally bizarre; they are round (Fig. 11) or oval (Fig. 12) although some may have unusual shapes (Figs. 13 to 15). Their outlines can be smooth (Figs. 12, 14, and 15) or knobby (Figs. 11, 16 to 19). On the whole the carcinomatous clusters are not as compact (Figs. 11 and 16) as the benign clusters of

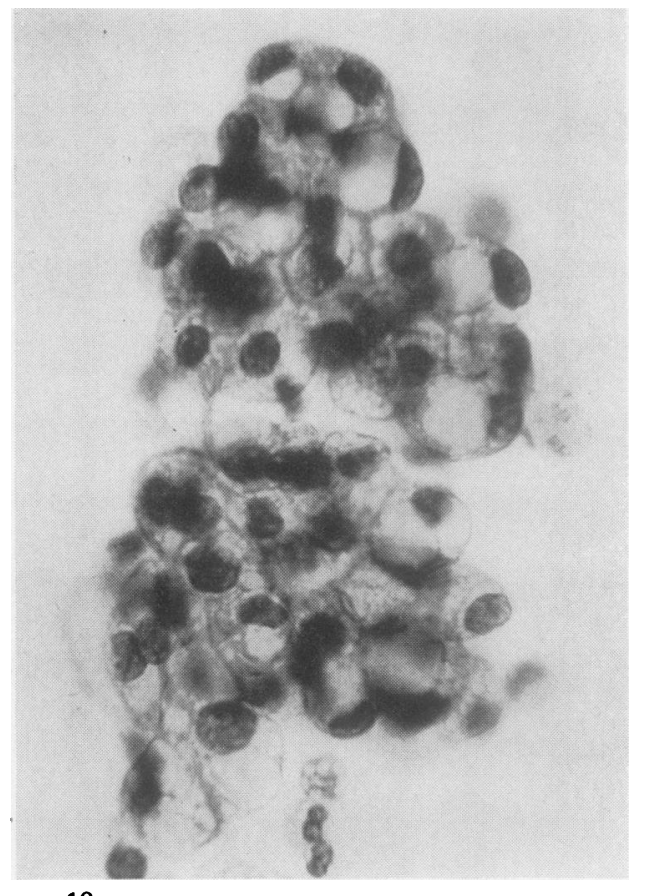

FIG. 18

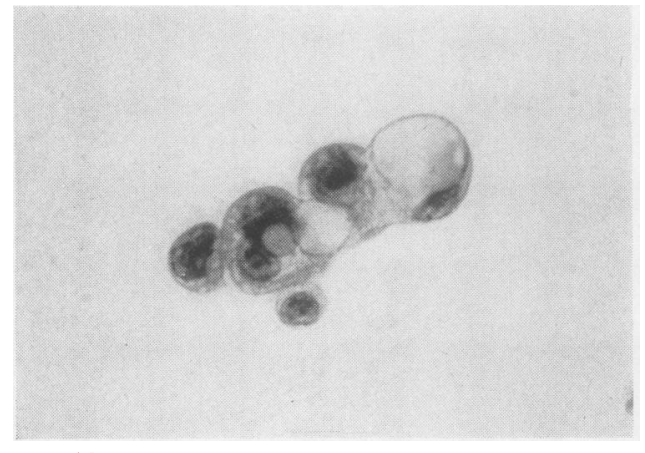

FIG. 19

asthmatics nor are they as likely to show peripheraff palisading; yet exceptions may be found (Fig. 15) The degree of vacuolation of the two types of cluste is similar (Figs. 17 to 19) but none of the carcino matous clusters ever showed cilia.

The nuclei provide important points of difference? carcinomatous nuclei are usually larger, their size and shape vary more within a given cluster, their nuclear boundaries tend to be heavier (Figs. 11, 12 and 16), and their chromatin pattern is more irregulai (Figs. 11, 12, 16, and 18). Mitotic figures are occasionally seen (Fig. 13) and nucleoli are ofter large and prominent (Figs. 11, 12, and 16). 


\section{DISCUSSION}

The finding of large compact clusters of columnar epithelial cells in smears of sputum of a patient with bronchial asthma may result in a false-positive report, and the consequences of such an error may obviously be quite serious (Philps, 1956). To avoid this cytodiagnostic pitfall is not always a simple matter; it often requires a nicety of judgment to distinguish between benign asthmatic and adenocarcinomatous clusters. Although they are not constant, the most important distinguishing features of clusters in asthmatics' sputa are the fairly uniform size and shape of the nuclei, the neat peripheral palisading of cells, and the presence of cilia. Many of the asthmatics' clusters may lack cilia; however, if they can be found, they signify benignancy, for we have never seen cilia on an adenocarcinoma cell.

Apart from the morphology of the clusters, there are extraneous observations which may help to prevent a wrong diagnosis: a history of asthma or the finding of many eosinophil leucocytes in the sputum should alert the cytologist. Curschmann's spirals were not too helpful since they were frequently found in the sputa of patients who were not asthmatics. Charcot-Leyden crystals were difficult to see without polarized light but when they were found they were always accompanied by numerous eosinophil leucocytes.

Excessive shedding of the mucosa of the lower respiratory tract occurs almost exclusively in asthmatics (Naylor, 1962) and, therefore, the large clusters of columnar epithelial cells are seldom encountered in the sputa produced in other respiratory diseases. Occasionally we have found similar clusters in the sputa of patients with bronchiectasis or chronic bronchitis but in these conditions they have never been numerous. Shedding of the mucosa is greatest during an attack of asthma, but it may persist, due to residual pathological changes, for at least several days after the attack subsides. It is, therefore, during and shortly after attacks of asthma that errors in cytodiagnosis may occur.

By far the most important factor in preventing a false-positive diagnosis is the awareness that the sputum of an asthmatic patient is likely to contain these unusual clusters of columnar epithelial cells. It is hoped that by drawing attention to this phenomenon some diagnostic errors may be prevented.

We wish to acknowledge with thanks the aid given to this investigation by grant $\mathrm{T}-28 \mathrm{E}$ from the American Cancer Society, Inc., New York. Also, we are most grateful to Miss Emma Lou Bridges, of the Papanicolaou Cancer Research Institute at Miami, for her valued criticism of the manuscript.

Figures 4 and 5 are reproduced by permission of the Editor of Thorax.

\section{REFERENCES}

Bamforth, J. (1959). Practitioner, 182, 702.

Cohen, R. C., and Prentice, A. I. D. (1959). Tubercle (Edinb.), 40, 44.

Farber, S. M., Wood, D. A., Pharr, S. L., and Pierson, B. (1957). Dis. Chest, 31, 1.

Naylor, B. (1962). Thorax, 17, 69.

Philps, F. R. (1956). Brit. J. Cancer, 10, 24.

Vincent Memorial Hospital (1950). The Cytologic Diagnosis of Cancer, by the Staff of the Vincent Memorial Laboratory. Saunders, Philadelphia. 